\title{
Refractory Ependymoma
}

National Cancer Institute

\section{Source}

National Cancer Institute. Refractory Ependymoma. NCI Thesaurus. Code C147108.

Ependymoma that does not respond to treatment. 